\title{
Uncovered
}

\section{Diamond: a gem for micro-optics}

\section{G. Seniutinas ${ }^{1, *}$, E. Brasselet ${ }^{2}$, A. Balčytis ${ }^{3}$, C. David ${ }^{1}$, S. Juodkazis ${ }^{3,4}$}

${ }^{1}$ Paul Scherrer Institute, $\mathrm{CH}-5232$ Villigen-PSI, Switzerland

${ }^{2}$ University of Bordeaux, CNRS, Laboratoire Ondes et

Matière d'Aquitaine, 351 cours de la libération, F-33400 Talence,

France

${ }^{3}$ Centre for Micro-Photonics, Faculty of Science, Engineering and Technology, Swinburne University of Technology, Hawthorn, Victoria 3122, Australia

${ }^{4}$ Melbourne Centre for Nanofabrication, Australian National Fabrication Facility, 151 Wellington Road, Clayton, Victoria 3168, Australia

Seniutinas, G. (gediminas.seniutinas@psi.ch)

Photonics have developed greatly over the last several decades and brought about a variety of new concepts in communications, sensing, solar energy and many other fields. With the emerging applications of quantum technologies and all-optical data processing, the impact of photonics on technology is undeniable, as evidenced through the International Year of Light and Light-based Technologies in 2015.

One of the key issues in the field is miniaturization and integration of photonic components, to enable light control at the microscale. Advancements in various fabrication techniques facilitate downscaling of optical elements; however, their design concepts need to be elaborated to keep the performance at the microscale. Metasurfaces and form birefringence structures have proved to be highly efficient for engineering arbitrary light fields $[1,2]$. Even though optical functionalities in these cases can be endowed by structuring materials on the subwavelength scale, the performance of components still highly depends on their material properties. Low absorption, high refractive index, high threshold of radiation damage, thermal stability, and high thermal conductivity are greatly desired material features to reduce

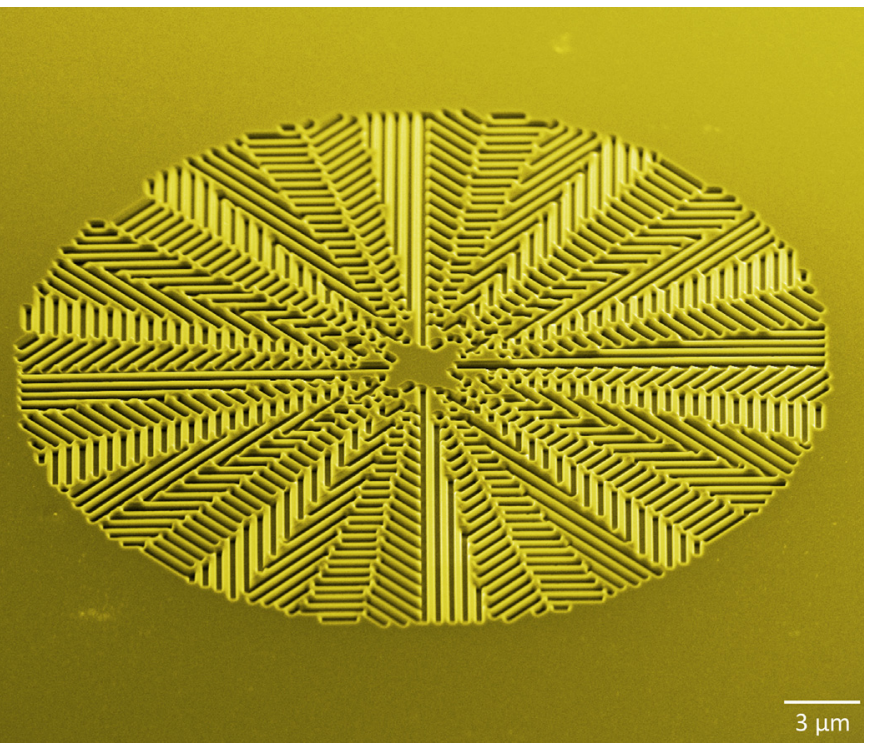

losses and assure stable performance over a broad range of operation conditions. All these properties are well combined in a material that has been admired for centuries - diamond. Being extraordinarily hard and chemically inert, it withstands harsh environments and aging. The large bandgap makes diamond appear crystal clear and highly transparent from the UV to the far infrared bands. In addition, high refractive index at visible wavelengths and ultimate thermal conductivity set diamond apart from other available materials and make it compelling for optics and photonics $[3,4]$. Nonetheless, diamond is naturally an almost isotropic material, thus birefringence and optical functionality must be created by shaping the material at the subwavelength scale.

Diamond optical elements based on form birefringence have been successfully demonstrated for the infrared band, where microscale patterning resolution is sufficient to accommodate longer wavelengths. High efficiency annular groove phase masks for infrared light attenuation in a vortex coronagraph were produced using nanoimprinting and reactive ion etching techniques [5]. Similar fabrication approaches were used making phase 
plates to control polarization in high-power $\mathrm{CO}_{2}$ lasers [6]. Also, diamond optics has been developed to manipulate high-intensity X-ray beams. It has been shown that diffractive elements made out of diamond can survive and keep their performance at high intensities even when used in a full beam of X-ray free electron lasers [7]. Despite many advantages, however, the use of diamond for micro-optics in the visible domain remained limited mainly because of the challenging fabrication. State-of-theart electron beam lithography offers sufficient patterning resolution of resist masks. Still, high-resolution pattern transfer into diamond is complicated due to high aspect ratio structures required to induce necessary phase shift between different polarization states of the incident light. Nonetheless, a robust nanofabrication process involving electron beam lithography and oxygen plasma etching has been recently developed to make $\mathrm{X}$-ray diffraction gratings [8]. We use a similar process to make subwavelength structures and establish micro-optical elements operating throughout the entire visible spectrum. We also investigated direct focused ion beam writing to mill desired patterns directly into the diamond. Both techniques allow for production of diamond micro-optics.

Due to the high refractive index of diamond, optical elements can be just a few micrometers in thickness, thus in our demonstration we used $\sim 5$ - $\mu$ m-thick chemical vapor deposition grown diamond membranes. Illustration of beam shaping capabilities of subwavelength-structured diamond micro-optical elements is made in the context of the generation of light beams carrying an arbitrary amount of orbital angular momentum per photon, so-called vortex beams. Such beams find a variety of applications, for instance in optomechanics, optical communications, optical imaging, and quantum optics. These optical components rely on imprinting an azimuthal phase profile to an incident light beam via the geometrical features of space-variant subwavelength gratings. We used discrete set of domains that act as microscale birefringent half-wave plates for the considered wavelength, whose individual optical axis is prescribed by the grating orientation. The domains were arranged by following design rules described in Ref. [9]. The design exploits the coupling between the polarization and spatial degrees of freedom of light, which refers to the spin-orbit interaction of light. In practice, an incident circularly polarized Gaussian beam is converted into a vortex beam carrying an orbital angular momentum defined by the structure of the micro-optical element and the handedness of the incident polarization state. An example of such an element designed to operate at $1-\mu \mathrm{m}$ wavelength is featured on the cover of this issue of Materials Today. The image shows scanning electron micrograph of a component enabling the generation of optical vortex beams with topological charge of 10 (i.e., the phase is exploring $20 \pi$ over one full turn). The extension to any other wavelength is readily achieved by design, adapting the grating pitch, depth, and filling factor to the need. Our current diamond nanopatterning processes enable fabrication of high aspect ratio gratings down to sub-100-nm period and opens up practical implementations of diamond micro-optics in a variety of applications.

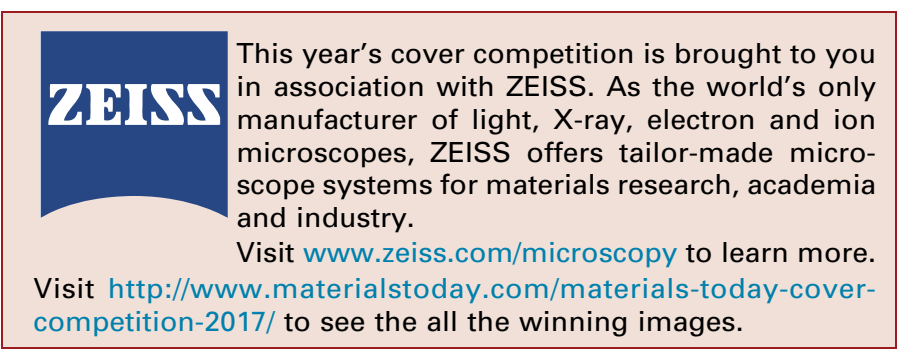

\section{Acknowledgments}

GS and CD acknowledge funding from the EU-H2020 Research and Innovation Program under Grant Agreement No. 654360 NFFA-Europe. This work was performed in part at the Melbourne Centre for Nanofabrication (MCN) in the Victorian Node of the Australian National Fabrication Facility (ANFF) and partly supported by the Australian Research Council DP130101205 grant.

\section{Further reading}

[1] N. Yu, F. Capasso, Nat. Mater. 13 (2014) 139-150.

[2] P. Genevet et al., Optica 4 (2017) 139-152.

[3] E. Pace et al., Diamond Relat. Mater. 10 (2001) 736-743.

[4] I. Aharonovich et al., Nat. Photon. 5 (2011) 397-405.

[5] P. Forsberg et al., Proc. SPIE 9151 (2014) 915119.

[6] P. Forsberg et al., Opt. Mater. Express 6 (2016) 262717.

[7] C. David et al., Sci. Rep. 1 (2011) 57.

[8] M. Makita et al., Microelectron. Eng. 176 (2017) 75-78.

[9] D. Hakobyan et al., Adv. Opt. Mater. 4 (2016) 306-312. 